\title{
Isolated Hydatid Cyst of the Kidney
}

\author{
Fatehi E. Elzein ${ }^{\mathrm{a}, \mathrm{d}}$, Abdullah Aljaberi ${ }^{\mathrm{a}}$, Shuaa Asiri ${ }^{\mathrm{b}}$, Abdullah Alghamdic
}

\begin{abstract}
Echinococcal cyst commonly involves the liver and the lungs. Renal hydatid cyst is unusual and tends to occur in association with other organ involvement. Isolated hydatid cyst is extremely rare and can mimic renal tumors. Hence, this diagnosis should be entertained in patients from endemic areas with solitary renal lesions. Although eosinophilia is a suggestive criterion, its absence should not dissuade from pursuing this diagnosis. We here describe a patient with prolonged history of lumbar pain resulting from a large hydatid cyst treated by nephrectomy with a pre- and postoperative course of albendazole and praziquantel.
\end{abstract}

Keywords: Renal hydatid cyst; Echinococcal cyst; Nephrectomy; Diagnosis

\section{Introduction}

Cystic echinococcosis (CE) is a parasitic disease caused by the larval form of E. granulosus. Dogs are the principal definitive host while sheep are the most common intermediate host. Humans get accidentally infected by ingesting the worm's eggs in contaminated food and water but do not infect others. The liver is involved in almost $70 \%$ of the cases. Larvae that escape filtration by the liver involve the lungs in $25 \%$ of the patients [1]. Although renal hydatid is associated with other organ involvement in $2-4 \%$ of the cases, isolated renal echinococcosis is extremely rare being reported in $1.9 \%$ of cases $[2,3]$.

Manuscript accepted for publication December 28, 2015

aDivision of Infectious Diseases, Department of Medicine, Prince Sultan Military Medical City, Riyadh 11159, Saudi Arabia

${ }^{b}$ Histopathology Department, Prince Sultan Military Medical City, Riyadh 11159, Saudi Arabia

'Urology Department, Prince Sultan Military Medical City, Riyadh 11159, Saudi Arabia

${ }^{\mathrm{d} C}$ Corresponding Author: Fatehi Elzein, Division of Infectious Diseases, Department of Medicine, Prince Sultan Military Medical City, Riyadh 11159, Saudi Arabia. Email: fatehielzein@gmail.com

\section{Case Report}

A 65-year-old woman with DM and hypertension was admitted to hospital with 8 years history of right lumbar pain. She used to live in the desert with strong history of contact with animals including dogs. She denied any urinary or other systemic symptoms. General examination was normal. Abdominal examination showed a $7 \times 6 \mathrm{~cm}$ paraumbilical hernia. US abdomen showed a complex cystic structure measuring $20 \times$ $9.4 \mathrm{~cm}$ occupying the right renal region that revealed multiple septations, coiled membranes and internal debris. Several internal small cysts are seen, features of which were consistent with hydatid cyst (Fig. 1, 2). Similarly, MRI disclosed a huge cystic lesion involving the upper and mid pole of the right kidney producing anterior displacement of the liver and pancreas with a thin T2 hypointense wall. There is a long smoothly outlined linear structure within the cyst, which includes multiple tiny cysts, likely representing an infolded ruptured wall of a hydatid cyst (Fig. 3). CT scan of the abdomen, chest and pelvis confirmed a giant hydatid cyst in the right kidney with no evidence of other cysts in the liver or the lungs (Fig. 4). The eosinophil count was normal both before and after surgery. Se-

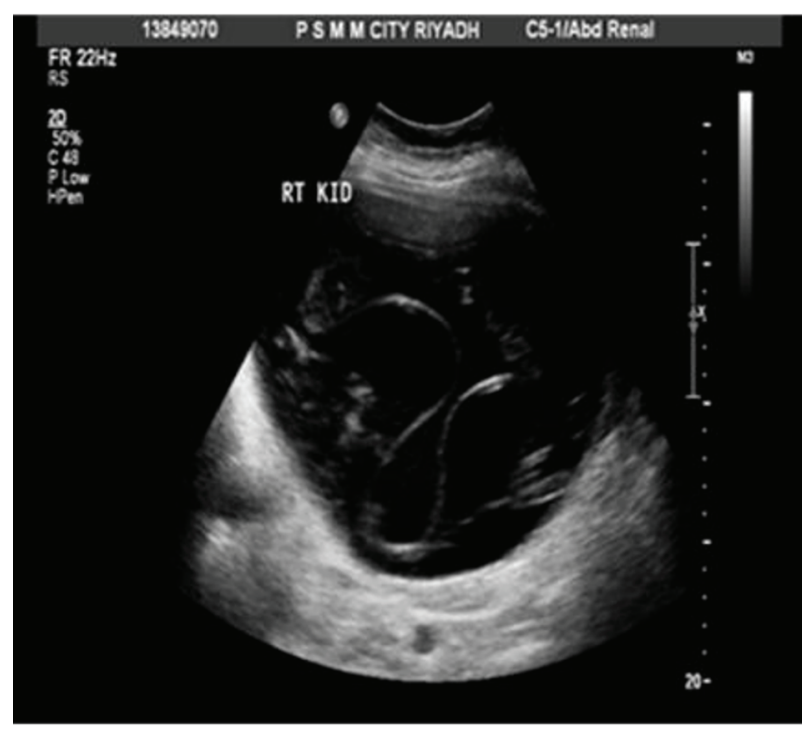

Figure 1. US abdomen showing right renal hydatid cyst. 


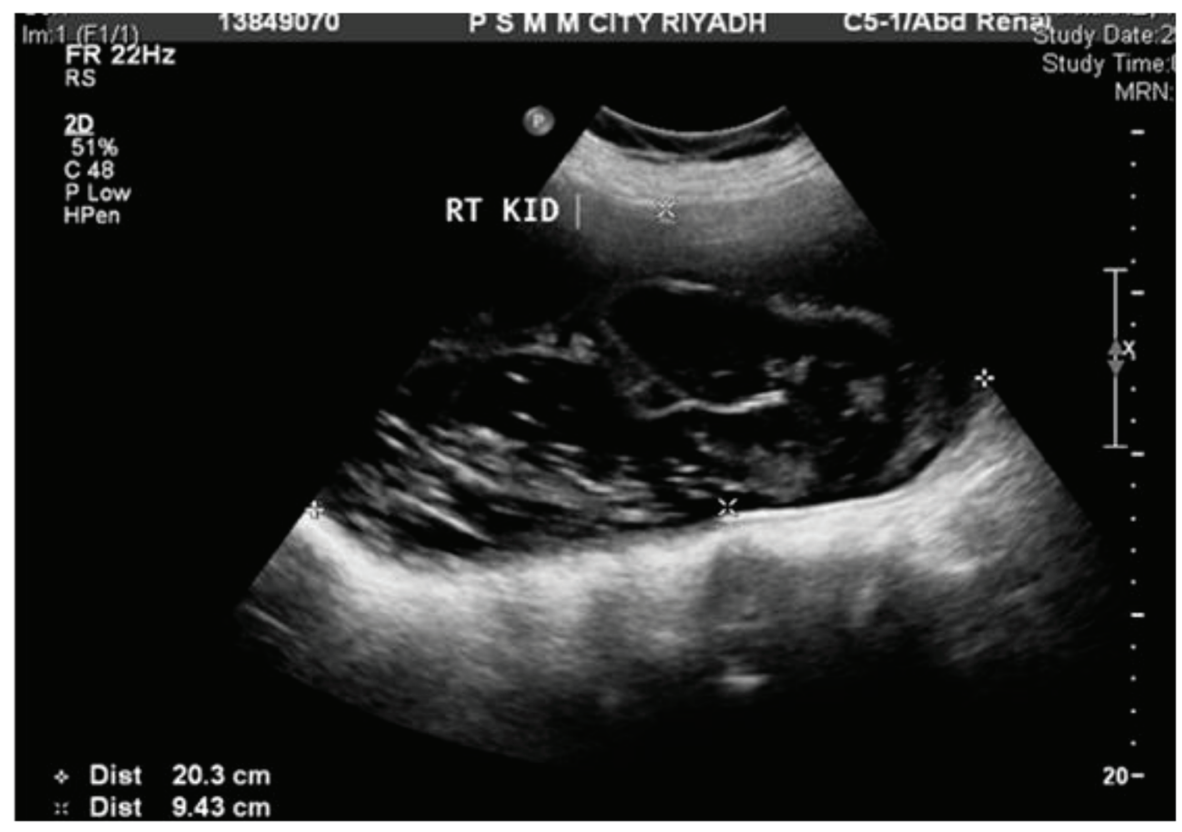

Figure 2. US abdomen.

rum creatine remained normal despite a right kidney contributing only $5 \%$ of the renal function on DMSA scan. Urine and aspirate of cyst fluid was repeatedly negative for hydaturia. IHA serology for hydatid was also negative.

Based on the radiological and exposure history, albendazole (400 mg twice daily) and praziquanel $600 \mathrm{mg}$ weekly were started for 4 weeks prior to surgery. Nephrectomy was done through retroperitoneal dissection of the kidney. The patient tolerated the procedure well with uneventful postop-

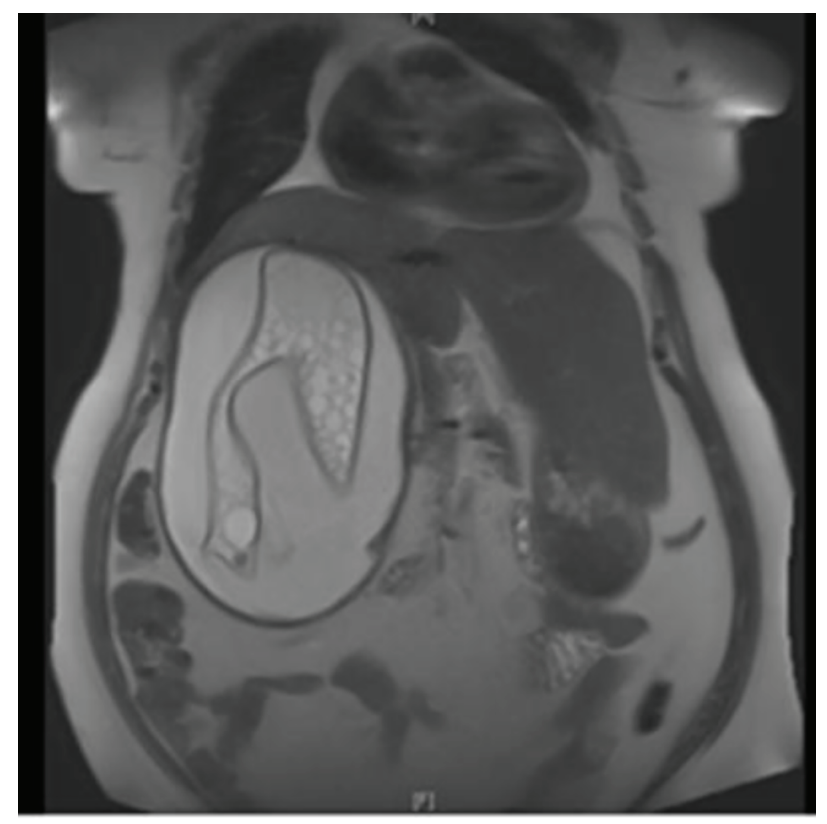

Figure 3. MRI of the kidneys - a huge hydatid cyst with ruptured true wall in the right kidney. erative course. Macroscopic appearance showed an intact unilocular cyst filled with multiple cysts of 0.5 up to $5 \mathrm{~cm}$ in size (Fig. 5). On microscopy, the cyst showed a characteristic wall's refractile eosinophilic lamination that is positive on PAS (Fig. 6). The uninvolved kidney shows chronic pyelonephritis, diabetic nephropathy and arteriosclerosis. She was discharged on albendazole $400 \mathrm{mg}$ BD for another 4 weeks.

\section{Discussion}

Renal hydatid disease is uncommon in Saudi Arabia. In a review of 20 years medical records of a tertiary care hospital in Riyadh, only six patients had isolated renal hydatid [4]. The cyst grows slowly and can remain asymptomatic for over 10 - 12 years [5]. The diagnosis is generally based on clinical findings, imaging techniques, and serology. Hydaturia, which is observed in $10-30 \%$ of the cases, is the only pathognomonic feature. On the other hand, proof of the presence of protoscoleces may be given by microscopic examination of the aspirated fluid and histology. Sensitivity of serum antibody detection using indirect hemagglutination, ELISA, or latex agglutination, ranges from 85 to $98 \%$ for liver cysts, $50-60 \%$ for lung cysts and 90-100\% for multiple organ cysts [6]. The IHA test is positive in about $75 \%$ of cases. As a result, a negative test, as in our patient, does not rule out the diagnosis [7]. Serology may be beneficial though, to exclude recurrence following surgery.

Ultrasound (US) examination is the basis of CE diagnosis in abdominal locations. Standardized classification based on US that could allow grouping of the cysts into active (CE1 and 2), transitional (CE3) and inactive (CE4 and 5), was developed by the WHO in 2003 [8]. Thus US may influence the choice of treatment. On the other hand, the location and depth of a cyst, 


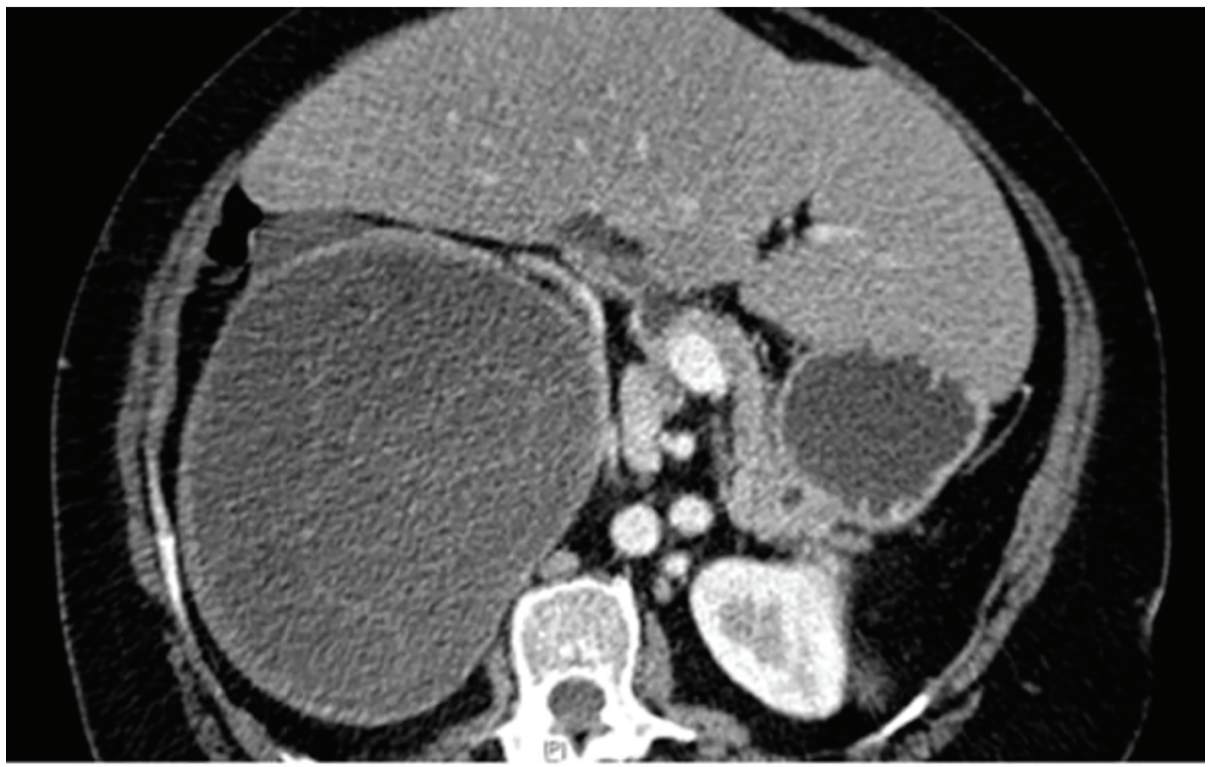

Figure 4. CT scan abdomen - huge hydatid cyst in the right kidney.

presence of daughter cysts and exogenous cysts tend to be seen more clearly on a CT scan [9]. Computed tomography (CT), and magnetic resonance imaging (MRI), with one T2-weighted imaging sequence are of particular value in subdiaphragmatic location, disseminated disease, extra-abdominal location and pre-surgical evaluation. MRI is preferred over CT due to its better visualization of liquid areas within the matrix $[10,11]$.

The treatment of renal hydatid disease is usually surgical. Medical treatment is customarily indicated before surgery to avoid relapses and to decrease the cyst wall tension, thus reducing the risk of spillage intra-operatively and associated

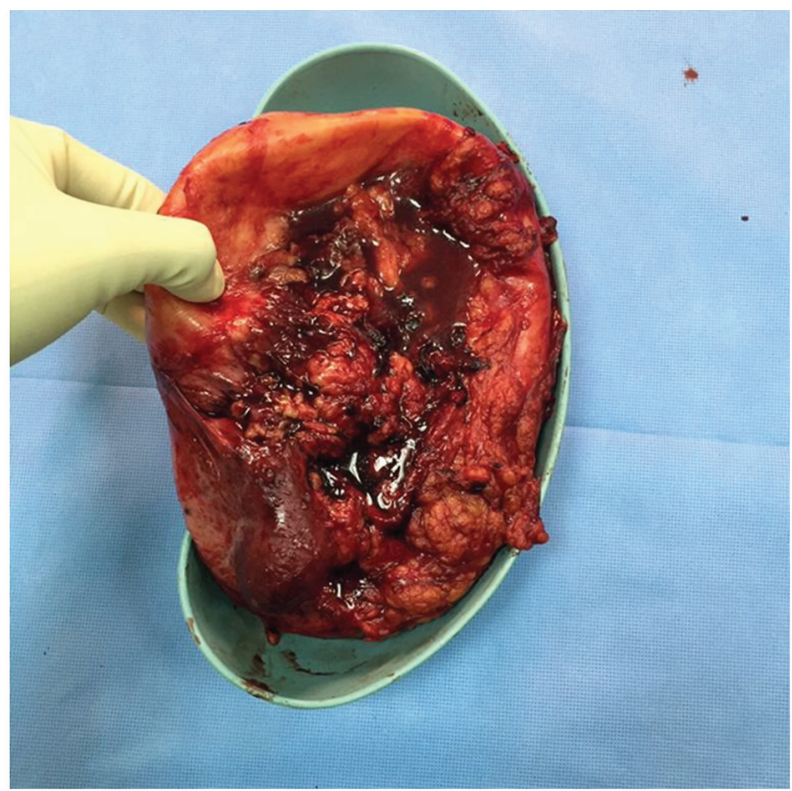

Figure 5. Removed (R) kidney gross pathology: an intact $21.0 \times 16.0 \times$ $10.0 \mathrm{~cm}$ unilocular cyst of the resected kidney. anaphylaxis. Moreover, medical treatment is the sole therapeutic option in disseminated hydatidosis. Albendazole with praziquantel combination demonstrated significant synergistic activity against Echinococcus spp. [12, 13]. A number of clinicians continue to use albendazole in cyclic interrupted courses. There are no trials that compare continuous albendazole with interrupted courses but, as albendazole blocks glucose uptake by the parasite, an interrupted course may allow the parasite to restock, thus reducing drug efficacy [14]. In addition to boosting intracystic albendazole levels, praziquantel acts as a protoscolicide to prevent disseminated disease following cyst leakage. Different regimens are used for praziquantel; however, an expert consensus recommends $60 \mathrm{mg} / \mathrm{kg}$ to be given weekly [6]. While percutaneous aspiration injection and respiration (PAIR) is undertaken in a majority of hepatic cysts $(54.1 \%)$, only $0.54 \%$ renal hydatid underwent percutaneous aspiration. Kidney sparing hydatid cyst removal (cystectomy with

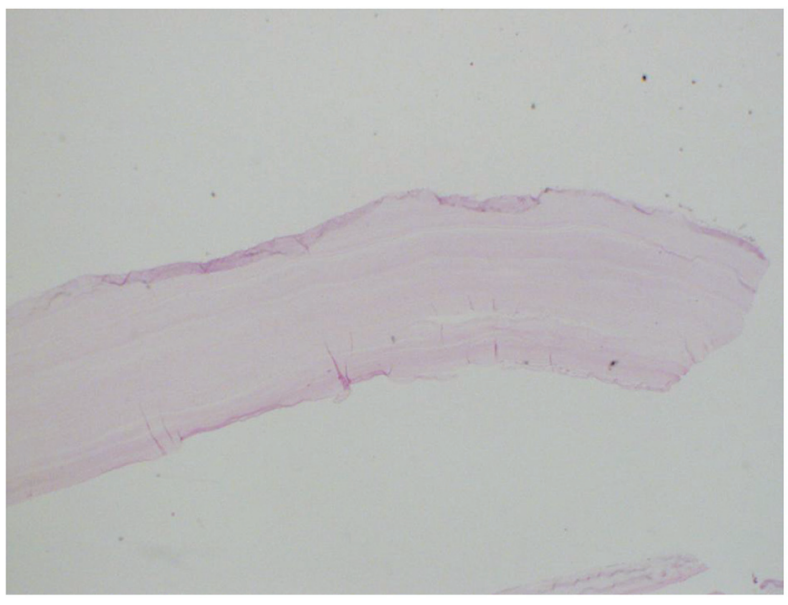

Figure 6. Histopathology of resected hydatid "echinococcal" cyst. 
pericystectomy) is possible in most cases (75\%). Conversely, nephrectomy remains the treatment of choice for complicated renal hydatid cysts and completely destroyed kidney where partial removal can lead to relapse and incomplete cure [2].

Hydatid cyst can masquerade as renal cell carcinoma. Although extremely rare, renal hydatid should be suspected and the diagnosis pursued in patients from endemic areas presenting with solitary kidney mass. Combined medical and parenchyma-preserving surgery is usually successful. In a few patients with non-functioning kidneys, total nephrectomy may be the only available option.

\section{References}

1. Pedrosa I, Saiz A, Arrazola J, Ferreiros J, Pedrosa CS. Hydatid disease: radiologic and pathologic features and complications. Radiographics. 2000;20(3):795-817.

2. Zmerli S, Ayed M, Horchani A, Chami I, El Ouakdi M, Ben Slama MR. Hydatid cyst of the kidney: diagnosis and treatment. World J Surg. 2001;25(1):68-74.

3. Zayed AM, Abdel-Rahman AH. Isolated hydatid cyst of the kidney. Diagn Surg Treat AJU. 2005;3:6-12.

4. Mokhtar AA, Sayyah AA, Al-Hindi H, Seyam RM, Khudair WA. Isolated renal hydatid disease in a non-endemic country: a single centre experience. Can Urol Assoc J. 2012;6(6):E224-229.

5. Eckert J, Deplazes P. Biological, epidemiological, and clinical aspects of echinococcosis, a zoonosis of increasing concern. Clin Microbiol Rev. 2004;17(1):107-135.

6. Brunetti E, Kern P, Vuitton DA. Expert consensus for the diagnosis and treatment of cystic and alveolar echinococ- cosis in humans. Acta Trop. 2010;114(1):1-16.

7. Shetty SD, al-Saigh AA, Ibrahim AI, Malatani T, Patil KP. Hydatid disease of the urinary tract: evaluation of diagnostic methods. Br J Urol. 1992;69(5):476-480.

8. International classification of ultrasound images in cystic echinococcosis for application in clinical and field epidemiological settings. Acta Trop. 2003;85(2):253-261.

9. Sayek I, Tirnaksiz MB, Dogan R. Cystic hydatid disease: current trends in diagnosis and management. Surg Today. 2004;34(12):987-996.

10. Hosch W, Junghanss T, Stojkovic M, Brunetti E, Heye T, Kauffmann GW, Hull WE. Metabolic viability assessment of cystic echinococcosis using high-field 1H MRS of cyst contents. NMR Biomed. 2008;21(7):734-754.

11. Junghanss T, da Silva AM, Horton J, Chiodini PL, Brunetti E. Clinical management of cystic echinococcosis: state of the art, problems, and perspectives. Am J Trop Med Hyg. 2008;79(3):301-311.

12. Cobo F, Yarnoz C, Sesma B, Fraile P, Aizcorbe M, Trujillo R, Diaz-de-Liano A, et al. Albendazole plus praziquantel versus albendazole alone as a pre-operative treatment in intra-abdominal hydatisosis caused by Echinococcus granulosus. Trop Med Int Health. 1998;3(6):462-466.

13. Alvela-Suarez L, Velasco-Tirado V, Belhassen-Garcia M, Novo-Veleiro I, Pardo-Lledias J, Romero-Alegria A, Perez del Villar L, et al. Safety of the combined use of praziquantel and albendazole in the treatment of human hydatid disease. Am J Trop Med Hyg. 2014;90(5):819822.

14. Nabarro LE, Amin Z, Chiodini PL. Current management of cystic echinococcosis: a survey of specialist practice. Clin Infect Dis. 2015;60(5):721-728. 